\title{
Correlation between seismicity and barometric tidal exalting
}

\author{
D. N. Arabelos ${ }^{1}$, G. Asteriadis ${ }^{1}$, A. Bloutsos ${ }^{2}$, M. E. Contadakis ${ }^{1}$, and S. D. Spatalas ${ }^{1}$ \\ ${ }^{1}$ Department of Geodesy and Surveying, Aristotle University of Thessaloniki, 54124, Thessaloniki, Greece \\ ${ }^{2}$ Department of Meteorology and Climatology, Aristotle University of Thessaloniki, 54124, Thessaloniki, Greece
}

Received: 6 June 2008 - Revised: 4 September 2008 - Accepted: 4 September 2008 - Published: 21 October 2008

\begin{abstract}
Changes of barometric pressure in the area of Thessaloniki in Northern Greece were studied by analyzing a sample of 31 years of hourly measurements. The results of this analysis on the periodicities of tidal components are expressed in terms of amplitude and phases variability. An earlier investigation revealed a detectable correlation between the exalting of the amplitude parameters of the tidal waves with strong seismic events. A problem of this work was that we had compared the tidal parameters resulting from the analysis of data covering the period of one year with instantaneous seismic events, although the earthquake is the final result of a tectonic process of the upper lithosphere. Consequently, in order to increase the resolution of our method we had analyzed our data in groups of 3-months extent and the resulted amplitudes were compared with seismicity index for corresponding time periods. A stronger correlation was found in the last case. However, the estimation of tidal parameters in this case was restricted to short period (from one day down to eight hours) constituents. Therefore, a new analysis was performed, retaining the one-year length of each data block but shifting the one year window by steps of three months from the beginning to the end of the 31 years period. This way, we are able to estimate again tidal parameters ranging from periods of one year (Sa) down to eight hours (M3). The resulting correlation between these tidal parameters with a cumulative seismicity index for corresponding time intervals was remarkably increased.
\end{abstract}

\section{Introduction}

It is broadly accepted and well documented that a number of physical phenomena and respectively a number of physical parameters present unusual behavior during the earth-

Correspondence to: D. N. Arabelos (arab@eng.auth.gr) quake preparation period as well as during the earthquake itself (e.g. Rikitake, 1981; Hayakawa, 1999; Hayakawa and Molchanov, 2002). The understanding of the intriguing way in which these phenomena are connected with the tectonic activity through the lithosphere-atmosphere-ionosphere coupling, is a challenging task by itself. In addition, due to the vast cost in lives and in social economy of a potential strong earthquake it has become imperative to investigate these phenomena in order to render any meaningful information about their connection with disastrous events and use the acquired knowledge for the effective mitigation of seismic hazard. Apart of the pure seismological research, a vast amount of research is currently carried out on a number of observables connected with these phenomena. A lot of atmospheric physical parameters i.e. density, ionospheric ion content, electromagnetic field, EM transmissivity etc., varies in a mutual interaction with lithospheric and ionospheric variations (e.g. Hayakawa et al., 1996; Silina et al., 2001; Mareev et al., 2002; Biagi et al., 2003; Molchanov et al., 2003, 2004; Plotkin, 2003). It could be used to detect extreme lithospheric variations, like the ones related to earthquakes. That requires a careful and very often sophisticated analysis to remove the inherent noise.

Atmospheric tides, excited by the periodically varying luni-solar gravitational attraction and heating by the Sun due to the earth revolution, subject to the influence of parameters affecting the thermal exciter (atmospheric temperature, water vapor content, ozone content, molecular viscosity and conductivity, ion content and ion drag of the ionosphere) as well as of parameters affecting the gravitational exciter (atmospheric density, topography and near surface crustal inhomogeneities) are well studied during the last two centuries. An extensive presentation of the state of the art up to the recent years is given by Chapman and Lindzen (1970), while an extensive analysis of the diurnal and semi-diurnal thermogravitational solar tide of the earth atmosphere in the area of Greece, was performed by Bartzokas (1989) and Bartzokas et al. (1995).

Published by Copernicus Publications on behalf of the European Geosciences Union. 
Atmospheric tides in the area of Thessaloniki were earlier studied by analyzing an 11 years sample of hourly barometric measurements (Arabelos et al., 1997). In an earlier paper by Biagi et al. (2003) the presence of tidal harmonics in the spectral content of three LF radio signals was reported indicating that atmospheric process with tidal periodicities are one of the factors affecting the propagation of LF radio waves. In addition they reported anomalous increase in the intensity of the radio signals which was propagated over the epicenter of a seismic sequence in Slovenia during the last month before the beginning of the sequence. They suggested that preseismic exalting of the atmospheric tides, produced by a gravity decrease due to fluid diffusion in a broad area around the epicenter, was responsible for the radio signal exalting. In a recent paper, Molchanov et al. (2004) present a general concept of mechanisms of preseismic phenomena in the atmosphere and ionosphere. Among them, they concluded that atmospheric perturbation of temperature and density could follow preseismic hot water/gas release near the ground surface. On the other hand, atmospheric tides depend on the atmospheric temperature and density (Chapman and Lindzen, 1970). Thus, if this concept is true, an alteration of the atmospheric tides, or at least of some constituent of them, is to be expected in the area of increased seismic activity around the time of its occurrence. These facts render the hope that observables related to atmospheric tides may probably be used as earthquake precursory phenomena (Gorbaticov et al., 1999).

Thessaloniki, being $30 \mathrm{~km}$ southeastern of the very active seismic area of Volvi and Langada lakes, is located under the threat of a potentially large earthquake. Therefore, a program for the investigation of a possible correlation of the atmospheric tides parameters with seismic activity in the area of Thessaloniki was initiated. The first results of this investigation (Arabelos et al., 2003, 2004) indicate a correlation of the annual variation of some wave groups with the seismic activity around Thessaloniki. This was shown by plotting the variations of the tidal amplitude of these wave groups together with individual earthquakes occurred around the test area.

In the present paper we investigate the variations of the tidal parameters of the barometric pressure in relation with the seismic activity in an extended area around Thessaloniki. A cross-correlation analysis of the tidal amplitude variations of certain wave groups with the seismicity of the area resulted in a quantitative consideration of this relation. The choice of the tidal waves considered is this work was based on their signal to noise ratio, regardless of their origin. Note that the aim of this work was to investigate possible correlation between seismicity and barometric tidal exalting, without attempting explanation of this coupling. Furthermore, it should be mentioned that with its present low resolution our method could not be applicable to forecast occurrence of earthquakes.

\section{Observational material and model of normal potential}

In the present analysis the data sets used were the following:

\section{Model of tidal potential}

From the models of Normal Tidal Potential we chose that of Tamura (1987) which includes 1200 waves.

\section{Barometric pressure and temperature data for the area of Thessaloniki}

The atmospheric pressure has been recorded with the help of a membrane box barograph and corrected with the help of a mercury pipe barometer installed in the same place with the barograph. The accuracy of each measurement of pressure is $\pm 1 \mathrm{hPa}$. The data set includes 267984 hourly values of the barometric pressure covering the period from 1 January 1975 to 31 December 2005 (11 166 days totally). These values have been derived from analogue registrations in the Meteorological Station of the Department of Meteorology and Climatology of the Aristotle University of Thessaloniki. The data set was used for the determination of the atmospheric modulations.

The atmospheric temperature was recorded with the help of a bimetallic thermograph and a mercury thermometer. The precision of each measurement of temperature is $\pm 0.1^{\circ} \mathrm{C}$. The hourly values of the temperature for the same period as in the section on barometric pressure data have been used to estimate the correlation between atmospheric temperature close to the earth's surface and barometric pressure.

\section{Earthquake catalogue}

The catalogue of the Geodynamic Institute/National Observatory of Athens was used, including 43128 earthquakes from 1964 up to now, occurred in the area bounded by $33.47^{\circ} \leq \varphi \leq 42.20^{\circ}, 16.26^{\circ} \leq \lambda \leq 30.36^{\circ}$. The local magnitude of these events varies from $M_{L}=1.4$ to $M_{L}=7$. In the next this would be referred as NOA catalogue.

\section{Analysis of the barometric measurements and com- putation of a seismicity index}

In Arabelos et al. $(2003,2004)$ it was attempted to detect correlation between amplitude exalting of the barometric tidal constituents and earthquakes occurred close to the area of Thessaloniki. The method of tidal analysis of the barometric pressure used permits the detailed estimation of amplitude and phase difference of tidal waves having periods from one year to one quarter of a day. However, in order to estimate the tidal parameters of long period waves such as the annual solar waves (Sa), it is necessary to analyze measurements covering a period of at least one year. On the other hand, the 
information resulting from our method might be useful if the correlation is concerned with shorter period.

In the afore-mentioned earlier papers the estimated annual values of the amplitude of 22 wave groups, resulting from the analysis of hourly barometric measurements covering the period from 1975 to 2005 (31 years), were plotted together with the earthquakes with $M \geq 4$ occurred at the same period, up to a distance of $200 \mathrm{~km}$ from Thessaloniki. The figures produced in this way showed a significant correlation in the case of some groups such as Sa and Ssa, while in some other cases the situation was confusing.

In order to be able to evaluate this correlation, it was necessary to compare the changes of the amplitude of the tidal waves with a "seismicity index" such as the seismic energy $E$, according to

$\log _{10} E_{S}=a+b M_{S}$

where $a, b$ are constants and $M_{S}$ is the surface magnitude of the earthquake. Since the computed amplitude values of the tidal waves are practically annual mean values the comparison should carried out with the cumulative energy $K$ for corresponding time intervals

$K_{i}=\sum_{t_{1}}^{t_{2}}\left(E_{S}\right)_{\psi \leq R}$

where $\psi$ is the distance of the earthquake epicenter from the barometric station, $R$ is the maximum distance up to which earthquakes were taken into account and $t_{1}, t_{2}$ define the corresponding annual time interval $i$. The estimation of $R$ was based on some numerical experiments as it will be discussed later on. For the constants $a$ and $b$ of Eq. (1) the values 4.8 and 1.5 , respectively, were suggested by Gutenberg and Richter (1956). The value 4.8 for the coefficient $a$ was considered as overestimated by Choy and Boatwright (1995). They suggest the value $a=4.4$ resulting from a global set of 397 earthquakes. However, the choice of $a$ is not critical for our analysis, since Eq. (1) is linear. On the other hand, for the coefficient $b$ which is critical for our analysis there is a general agreement between the authors.

Since for the estimation of Eq. (1) the surface magnitude $M_{S}$ of the earthquakes is needed, it was necessary to transform the local magnitude $M_{L}$ included in the NOA catalogue to corresponding surface one. For this purpose the equation

$M_{S}=c+d M_{L}$,

was used, where $c, d$ constants. For the evaluation of Eq. (3) the values of $c=-3.35 \pm 0.263$ and $d=1.687 \pm 0.053$ were defined by Tzanis and Vallianatos (2003) after a sophisticated comparison of 1184 common events between 1 January 1978 and 30 April 2000, contained in the NOA- $M_{L}$ and the International Seismological Centre (ISC)- $M_{S}$ catalogues. Following the authors the two magnitude scales are apparently linearly correlated for $M_{S}>3$ and $M_{L}>3.4-3.5$. In a new attempt we tried to define the constants $c$ and $d$, using the available common events of NOA- $M_{L}$ and ISC $M_{S}$, between 1 January 1978 and 31 December 2006. A number of 1416 common events were identified, allowing differences in time up to $8 \mathrm{~s}$ and up to 0.2 degrees in the latitude/longitude coordinates (not simultaneously at the same record). The linear regression model (Eq. 3) was used for the comparison of the magnitudes, resulting in the values $c=-1.5499, d=1.2565$ with a relatively low coefficient of determination $r^{2}=0.608$. In Table 1 the statistics of the differences $M_{S}^{\mathrm{ISC}}-M_{S}^{\mathrm{NOA}}$ is shown, where $M_{S}^{\mathrm{NOA}}=c+d M_{L}^{\mathrm{NOA}}$.

The statistics in both cases, either using the constants by Tzanis and Vallianatos (2003) or the computed in the present work are very close. The small differences shown in Table 1 are due to the fact that the constants by Tzanis and Vallianatos (2003) have been used practically to extrapolate the additional data used for the determination of the coefficients of the present work. However these results could not be considered satisfactory for this study. Therefore, we decided to avoid transformation of $M_{L}$ to $M_{S}$ and to substitute the radiated energy $E_{S}$ by a seismicity index (e.g. Tzanis and Vallianatos, 2003)

$\log _{10} \Omega=a+b M$,

where $a, b$ are constants and $M$ is the local magnitude of the earthquake. For the evaluation of Eq. (4) the values suggested by Gutenberg and Richter (1956), i.e. $a=4.8$ and $b=1.5$ were used for the reason discussed previously. The comparison with the computed annual mean values of the tidal waves was carried out with the cumulative values of the index $C$ for corresponding time intervals according to

$C_{i}=\sum_{t_{1}}^{t_{2}} \log _{10}(\Omega)_{\psi \leq R}$.

To estimate tidal parameters for periods ranging from half a year (Ssa) down to eight hours (M3) and simultaneously to keep a reasonable resolution, we performed the analysis of the barometric data in blocks, retaining the one year length of each block and using a shift of this window by a three months step from the beginning to the end of the time series. Note that performing the analysis in this way, smoothed amplitude changes were resulted instead of the actual ones. This was a necessary compromise though, for the method of analysis followed. Consequently, the summation from $t_{1}$ to $t_{2}$ in Eq. (5) was extended for corresponding intervals of one year. Values of $C$ computed in this way for the test area, Thessaloniki are shown in Fig. 1, in a form of bar charts. The values of $C$ were computed taking into account earthquakes with $M \geq 2$ (lower part) and $M \geq 4$ (upper part). Numerical experiments which will be described in Sect. 4 showed that earthquakes with $M<4$ are not correlated with changes of the barometric tides. The maximum $C$ in Fig. 1 is due to the seismic sequence of 21 shocks occurred in June 1978 at the seismic area of the village Stivos $(\approx 34 \mathrm{~km}$ east to Thessaloniki). The earthquake of $M=6$, occurred on 20 June 1978 
Table 1. Statistics of the differences between $M_{S}^{\mathrm{ISC}}$ and $M_{S}^{\mathrm{NOA}}$, based on the estimated constants $c$ and $d$ of Eq. (3).

\begin{tabular}{lrrrrr}
\hline Constants & No & $\begin{array}{r}\text { Mean } \\
\text { value }\end{array}$ & $\begin{array}{r}\text { Standard } \\
\text { deviation }\end{array}$ & $\begin{array}{r}\text { Minimum } \\
\text { difference }\end{array}$ & $\begin{array}{r}\text { Maximum } \\
\text { difference }\end{array}$ \\
\hline$c=-3.350, d=1.687$ & 1416 & -0.072 & 0.512 & -1.591 & 3.071 \\
$c=-1.485, d=1.242$ & 1416 & 0.000 & 0.471 & -1.353 & 2.950 \\
\hline
\end{tabular}
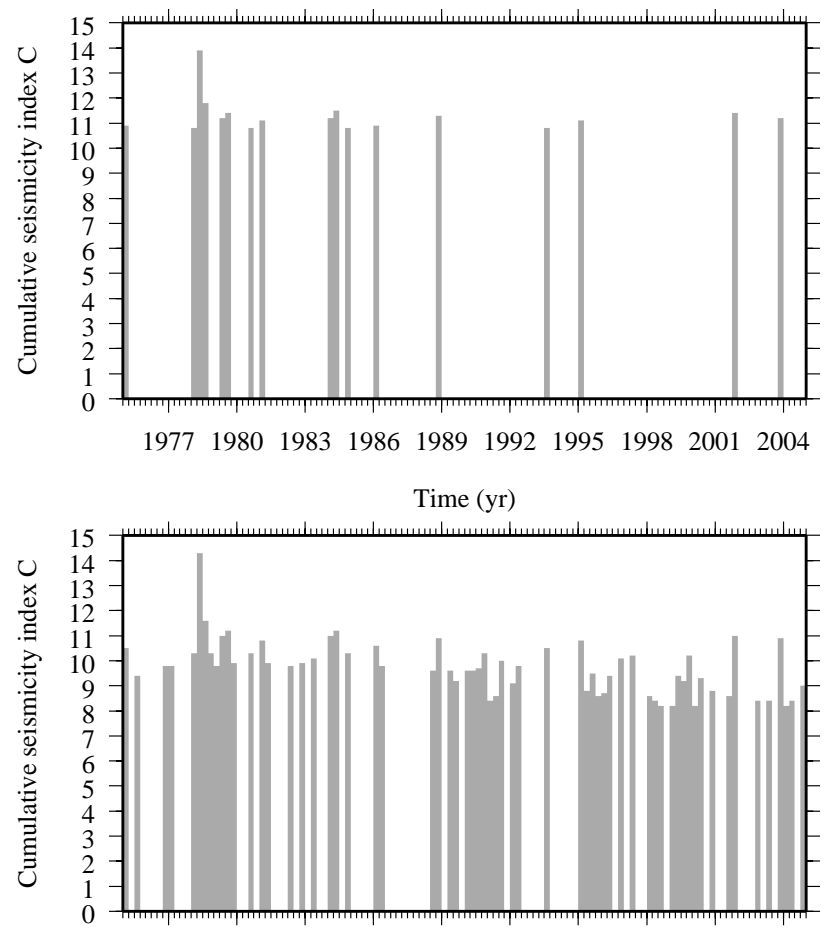

$\begin{array}{llllllllll}1977 & 1980 & 1983 & 1986 & 1989 & 1992 & 1995 & 1998 & 2001 & 2004\end{array}$

Time (yr)

Fig. 1. Cumulative values of $C$, computed for earthquakes up to a distance of $40 \mathrm{~km}$ from Thessaloniki taking into account earthquakes with $M \geq 2$ (lower part) or $M \geq 4$ (upper part).

belongs to this sequence. The smaller values of $C$ are due to rather isolated events ( 1 to 2 shocks).

Using the time series of hourly values of the barometric pressure with annual extent it is possible to estimate tidal parameters for periods of half a year (Ssa) down to eight hours. In order to exclude the case that the barometric tide disturbances are due to changes of the atmospheric temperature close to the earth's surface, the correlation between barometric tides and atmospheric temperature was estimated using a similar data set of hourly atmospheric temperature values. A linear regression model was used for this purpose. Then, the correlated part was subtracted from the original observation. In Fig. 2, the correlation of the barometric changes with the

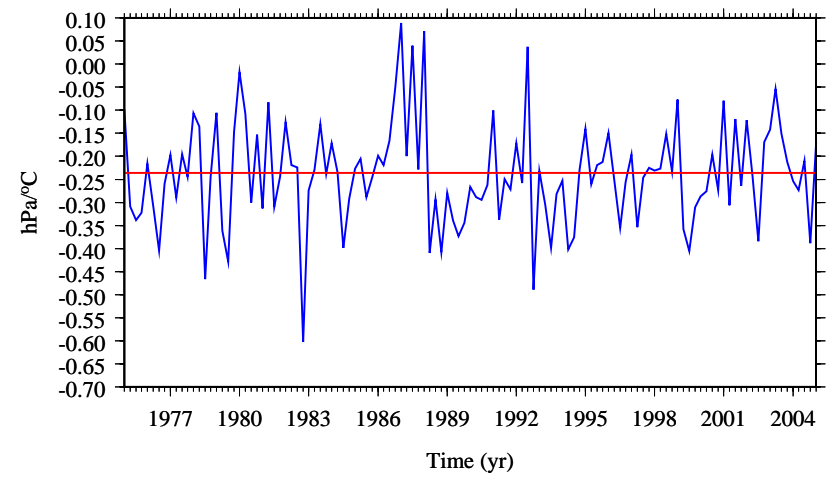

Fig. 2. Correlation between the barometric changes and atmospheric temperature computed for each annual group of observations separately (blue line). The red line corresponds to the correlation computed for the entire period of the 31 years.

atmospheric temperature is shown computed for each of the 121 groups of observations separately. The accuracy of this estimation ranges from 0.001 to $0.01 \mathrm{hPa} /{ }^{\circ} \mathrm{C}$. Tidal parameters were estimated for 18 tidal waves for each of the 121 groups of observations but only the parameters of 7 waves were used in the correlation analysis which will be described in the next section, since the signal to noise ratio was very weak (below 1.0 in average) for the rest. In Table 2, the mean, minimum and maximum of the 121 values of signal to noise ratio are shown for only these 7 tidal waves, which are the basis of our correlation analysis. These values are based on least square analysis. In Fig. 3 the changes of the tidal amplitude of Ssa and $\mathrm{Mm}$ are shown.

\section{Cross-correlation analysis}

The cross-correlation between the amplitude of the tidal waves designated by $A_{i}$ and the seismicity index $C_{i}$, was computed using the well known formula (e.g. Davis, 1973)

$$
r_{m}=\frac{n^{*} \sum A_{i} C_{i}-\sum A_{i} \sum C_{i}}{\left\{\left[n^{*} \sum A_{i}^{2}-\left(\sum A_{i}\right)^{2}\right]\left[n^{*} \sum C_{i}^{2}-\left(\sum C_{i}\right)^{2}\right]\right\}^{1 / 2}},
$$


Table 2. Mean, minimum and maximum values of signal to noise ratio for each tidal wave estimated for the 121 groups of observations based on least squares analysis.

\begin{tabular}{lcccc}
\hline Wave & Period (day) & Mean & Minimum & Maximum \\
\hline Ssa & 182.62 & 21.73 & 3.98 & 48.10 \\
Mm & 27.55 & 14.15 & 2.80 & 40.40 \\
Mf & 13.66 & 12.55 & 2.78 & 31.60 \\
Mtm & 9.13 & 8.47 & 1.00 & 25.60 \\
P1 & 1.00 & 4.65 & 0.10 & 10.34 \\
K1 & 1.00 & 4.69 & 0.60 & 11.30 \\
S2 & 0.5 & 5.43 & 3.50 & 8.30 \\
\hline
\end{tabular}

where $r_{m}$ the cross-correlation coefficient for match position $m$ and $n^{*}$ the number of overlapped positions between the two chains.

The significance of the cross-correlation coefficient can be assessed by the approximate test

$t=r_{m}\left(\frac{n^{*}-2}{1-r_{m}^{2}}\right)^{1 / 2}$,

which has $\left(n^{*}-2\right)$ degrees of freedom. This test was derived from a test for the significance of the correlation between two samples drawn from normal populations. The null hypothesis is that the correlation is zero. The algorithm used slides the first chain past the second one, computing successive values of $r_{m}$ and corresponding values of $t$ for overlapped segments of $n^{*} \geq 3$ because there are no degrees of freedom for $n^{*}<3$. In this work we present values of $r_{m}$ and $t$ only for the complete overlapping of the two chains under consideration.

The cross-correlation was computed taking into account earthquakes of different magnitude and in different distances from the barometric pressure station. More specifically, the seismicity index was computed taking into account (i) earthquakes with $M \geq 2, M \geq 3, M \geq 4$ and $M \geq 5$ and (ii) for all cases of (i), including for the computation of $C$ earthquakes up to distances ranging from 30 up to $250 \mathrm{~km}$ from the barometric pressure station. The correlation was computed ignoring quiet periods (with $C=0$ ). If these periods were included in the analysis the correlation coefficients will result in considerably reduced values. A plausible explanation of this could be that there are many other causes influencing the changes of the barometric pressure.

In Table 3 the statistical data of the cross-correlation analysis for seismicity index including earthquakes up to a radius $R=200 \mathrm{~km}$ from the barometric station are shown for the long period waves $S s a$, Mm, Mf and Mtm. The corresponding statistical data for the short period waves P1, K1 and $\mathrm{S} 2$ are shown in Table 4. Correlation coefficients marked with a $\bullet$ are passing the statistical test of significance. In both tables the critical values $t_{n^{*}-2}^{0.025}$ are shown in the last column. In Figs. 4 and 5 the changes of the correlation coefficient

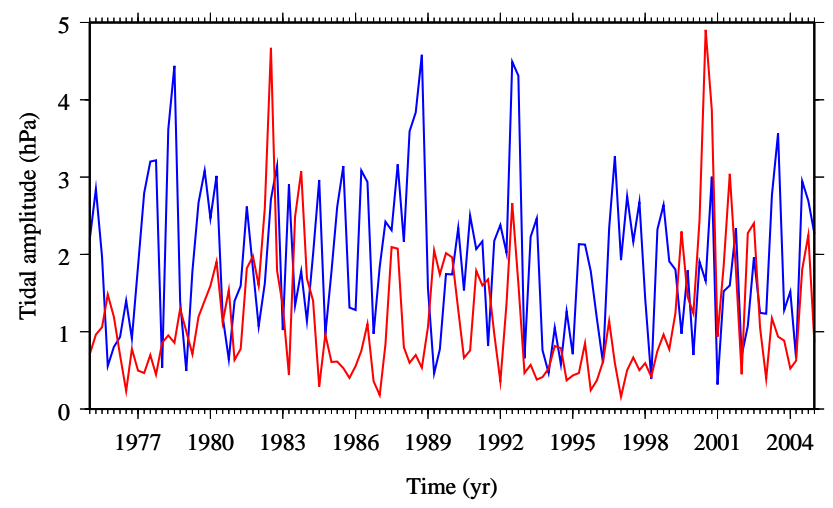

Fig. 3. Changes of the amplitude of Ssa (blue) and of Mm (red) of the 121 annual groups of hourly barometric observations.

with the distance up to $R=250 \mathrm{~km}$ are shown for the long and short period waves, respectively. Note that for $M \geq 5, C$ is computed starting from a collection radius of $70 \mathrm{~km}$, since there are very few events in shorter distances from Thessaloniki, during the entire period considered. Therefore, the distance scale for $M \geq 5$ is different than the corresponding in the other diagrams.

In Table 3 and Fig. 4 it is shown that for $M \geq 5$ considerable correlation coefficients are resulting for values of $C$ computed with a collection radius up to $150 \mathrm{~km}$ from the barometric pressure station. These correlation coefficients failed to pass the $t$-test for significance level equal to 0.05 . However, the degrees of freedom are not statistically sufficient up to a distance of $160 \mathrm{~km}$ from the barometric station. By increasing the collection radius the correlation becomes weaker and practically vanishes after the distance of about $150 \mathrm{~km}$. Furthermore, the correlation becomes weaker for $C$ including earthquakes with $M \geq 4, M \geq 3$ and $M \geq 2$, although larger values of $C$ resulted in these cases. From this point of view it might be concluded that distant or weak earthquakes are not correlated with the seismicity of the test area and consequently they behave as noise, decreasing in this way the overall correlation. Note that the correlation coefficients for Ssa are passing the $t$-test, for seismicity index computed from earthquakes with $M \geq 4, M \geq 3$ and $M \geq 2$ collected up to $150 \mathrm{~km}$ from the barometric station.

In Table 4 for the short period waves P1, K1 and S2 considerable values of correlation coefficients up to a distance of $200 \mathrm{~km}$ are shown for $M \geq 5$. For degrees of freedom from 1 to 3 the coefficients for P1 and K1 failed to pass the $t$-test. The situation was changed for the rest degrees (from 4 to 11). For $M \geq 4$ there is a rather rapid attenuation of the correlation coefficients for $\mathrm{P} 1$ and $\mathrm{K} 1$, although they are passing the test up to $R=90 \mathrm{~km}$. For $M \geq 3$ and $M \geq 2$ their correlation was almost vanished. The coefficients for $\mathrm{S} 2$ have considerable values almost for all distances $R$, for $M \geq 5, M \geq 4, M \geq 3$ and $M \geq 2$, passing the $t$-test from $\left(n^{*}-2\right) \geq 2$. Thus, the behavior 

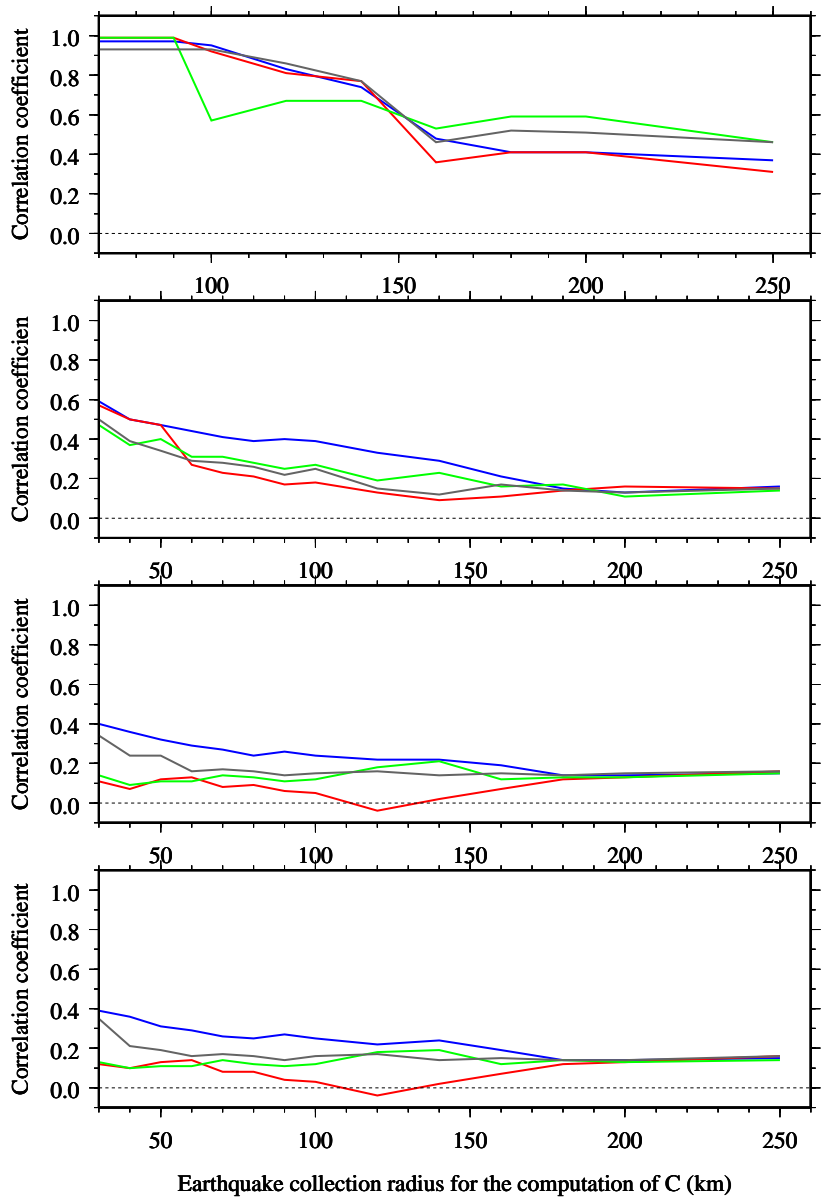

Fig. 4. Correlation coefficients between the amplitude of long period waves Ssa (blue), Mm (red), Mf (green) and Mtm (gray) and the seismicity index $C$. From the bottom to the top: $C$ computed including earthquakes with $M \geq 2, M \geq 3, M \geq 4$ and $M \geq 5$.

of the short period waves is promising for an increase of the resolution of this method, because the estimation of tidal parameters of the barometric pressure for the diurnal and semidiurnal waves is possible using time series of much shorter extend than those needed for the long period waves.

\section{Conclusion}

The analysis of the barometric tides in the area of Thessaloniki showed a clear correlation between the disturbances of the amplitude of some long period wave groups with the seismicity of the test area resulting from earthquakes with $M \geq 5$ up to distance of about $150 \mathrm{~km}$ from the barometric station. For earthquakes with $M \geq 4$ the correlation is rapidly decreasing and practically vanishes after a distance of about 50 to $60 \mathrm{~km}$ from the barometric station.

Considerable values of correlation are also observed in the case of some diurnal and semi-diurnal waves, with domi-
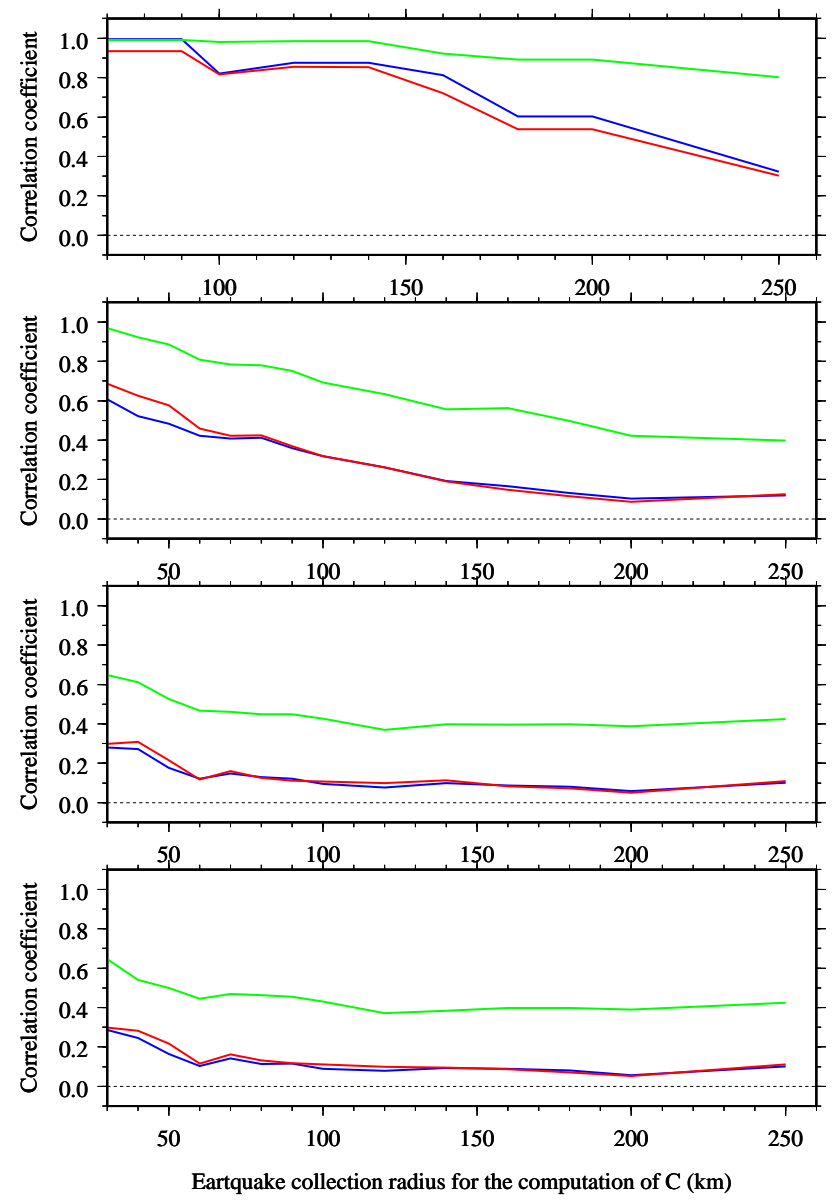

Fig. 5. Correlation coefficients between the amplitude of short period waves P1 (blue), K1 (red) and S2 (green) and the seismicity index $C$. From the bottom to the top: $C$ computed including earthquakes with $M \geq 2, M \geq 3, M \geq 4$ and $M \geq 5$.

nant the case of $\mathrm{S} 2$, where significant values of correlation resulted even for distances up to $200 \mathrm{~km}$ from the barometric station.

The correlation in general becomes weaker by increasing the radius of earthquakes collection for the computation of the seismicity index. On the other hand, the correlation becomes weaker as earthquakes with small magnitude are included in the computation of the seismicity index, although the seismicity index is increasing. This behavior allows us to conclude that distant (even strong) earthquakes or weak earthquakes (even very close to the test area) are not correlated with the tidal changes of the barometric pressure.

The study of the correlation between the seismicity and the barometric tidal exalting might be useful as a precursor if it could be possible to recognize the barometric tidal exalting using shorter time series of barometric observations. The behavior of the diurnal and semi-diurnal waves is promising for an increase of the resolution of this method. 
Table 3. Results of the cross-correlation analysis for the long period waves Ssa, Mm, Mf and Mtm. The index $m$ of $r$ denotes the number of terms matched, which in our case coincides with the number $N$ of $C>0$ in each chain. In addition $N$ must be at least 3 in order to fulfill the condition $n^{*} \geq 3$. The seismicity index $C$ was computed including earthquakes with $M \geq 5, M \geq 4, M \geq 3$ and $M \geq 2$. Correlation coefficients marked by a ${ }^{\bullet}$ are passing the $t$-test for significance level equal to 0.05 .

\begin{tabular}{|c|c|c|c|c|c|c|c|c|c|c|c|c|}
\hline \multirow[b]{2}{*}{ Magnitude } & \multirow[b]{2}{*}{$R(\mathrm{~km})$} & \multirow[b]{2}{*}{$m$} & \multirow[b]{2}{*}{$n^{*}-2$} & \multicolumn{2}{|c|}{ Ssa } & \multicolumn{2}{|c|}{$\mathrm{Mm}$} & \multicolumn{2}{|c|}{ Mf } & \multicolumn{2}{|c|}{ Mtm } & \multirow[b]{2}{*}{$t_{n^{*}-2}^{0.025}$} \\
\hline & & & & $r_{m}$ & $t$ & $r_{m}$ & $t$ & $r_{m}$ & $t$ & $r_{m}$ & $t$ & \\
\hline \multirow{13}{*}{$M \geq 5$} & 30 & 1 & - & - & - & - & - & - & - & - & - & \\
\hline & 40 & 1 & - & - & - & - & - & - & - & - & - & \\
\hline & 50 & 1 & - & - & - & - & - & - & - & - & - & \\
\hline & 60 & 2 & - & - & - & - & - & - & - & - & - & \\
\hline & 70 & 3 & 1 & 0.97 & 4.06 & 0.99 & 7.75 & 0.99 & 6.30 & 0.93 & 2.47 & 12.71 \\
\hline & 80 & 3 & 1 & 0.97 & 4.06 & 0.99 & 7.75 & 0.99 & 6.30 & 0.93 & 2.47 & 12.71 \\
\hline & 90 & 3 & 1 & 0.97 & 4.06 & 0.99 & 7.75 & 0.99 & 6.30 & 0.93 & 2.47 & 12.71 \\
\hline & 100 & 4 & 2 & 0.95 & 4.11 & 0.92 & 3.30 & 0.57 & 0.99 & 0.93 & 3.59 & 4.30 \\
\hline & 120 & 5 & 3 & 0.83 & 2.58 & 0.81 & 2.42 & 0.67 & 1.57 & 0.86 & 2.94 & 3.18 \\
\hline & 140 & 6 & 4 & 0.74 & 2.22 & 0.77 & 2.40 & 0.67 & 1.83 & 0.77 & 2.43 & 2.78 \\
\hline & 160 & 12 & 10 & 0.48 & 1.71 & 0.36 & 1.23 & 0.53 & 1.95 & 0.46 & 1.64 & 2.23 \\
\hline & 180 & 13 & 11 & 0.41 & 1.48 & 0.41 & 1.50 & $0.59^{\bullet}$ & 2.41 & 0.52 & 2.00 & 2.23 \\
\hline & 200 & 13 & 11 & 0.41 & 1.48 & 0.41 & 1.50 & $0.59^{\bullet}$ & 2.41 & 0.52 & 2.00 & 2.23 \\
\hline \multirow{13}{*}{$M \geq 4$} & 30 & 9 & 7 & 0.59 & 1.94 & 0.57 & 1.82 & 0.47 & 1.39 & 0.50 & 1.54 & 2.37 \\
\hline & 40 & 17 & 15 & $0.50^{\bullet}$ & 2.25 & $0.50^{\bullet}$ & 2.23 & 0.37 & 1.55 & 0.39 & 1.66 & 2.13 \\
\hline & 50 & 19 & 17 & $0.47^{\bullet}$ & 2.19 & $0.47^{\bullet}$ & 2.21 & 0.40 & 1.80 & 0.34 & 1.50 & 2.11 \\
\hline & 60 & 25 & 23 & $0.44^{\bullet}$ & 2.38 & 0.28 & 1.38 & 0.31 & 1.59 & 0.29 & 1.47 & 2.07 \\
\hline & 70 & 30 & 28 & $0.41^{\bullet}$ & 2.38 & 0.23 & 1.23 & 0.31 & 1.70 & 0.28 & 1.53 & 2.05 \\
\hline & 80 & 32 & 30 & $0.39^{\bullet}$ & 2.35 & 0.21 & 1.16 & 0.28 & 1.62 & 0.26 & 1.46 & 2.04 \\
\hline & 90 & 35 & 33 & $0.40^{\bullet}$ & 2.53 & 0.16 & 0.96 & 0.25 & 1.51 & 0.22 & 1.30 & 2.02 \\
\hline & 100 & 38 & 36 & $0.39^{\bullet}$ & 2.53 & 0.18 & 1.11 & 0.27 & 1.67 & 0.25 & 1.53 & 2.02 \\
\hline & 120 & 49 & 47 & $0.33^{\bullet}$ & 2.37 & 0.13 & 0.86 & 0.19 & 1.30 & 0.15 & 1.06 & 2.00 \\
\hline & 140 & 57 & 55 & $0.29^{\bullet}$ & 2.24 & 0.09 & 0.68 & 0.23 & 1.78 & 0.12 & 0.93 & 2.00 \\
\hline & 160 & 72 & 70 & 0.21 & 1.77 & 0.11 & 0.92 & 0.16 & 1.35 & 0.17 & 1.46 & 2.00 \\
\hline & 180 & 86 & 84 & 0.15 & 1.41 & 0.14 & 1.31 & 0.17 & 1.59 & 0.14 & 1.32 & 2.00 \\
\hline & 200 & 99 & 97 & 0.13 & 1.30 & 0.16 & 1.55 & 0.11 & 1.07 & 0.13 & 1.27 & 2.00 \\
\hline \multirow{13}{*}{$M \geq 3$} & 30 & 42 & 40 & $0.40^{\bullet}$ & 2.73 & 0.11 & 0.69 & 0.14 & 0.89 & $0.34^{\bullet}$ & 2.28 & 2.02 \\
\hline & 40 & 59 & 57 & $0.36^{\bullet}$ & 2.91 & 0.07 & 0.54 & 0.09 & 0.69 & 0.24 & 1.85 & 2.02 \\
\hline & 50 & 75 & 73 & $0.32^{\bullet}$ & 2.90 & 0.12 & 1.03 & 0.11 & 0.93 & $0.24^{\bullet}$ & 2.09 & 2.00 \\
\hline & 60 & 84 & 82 & $0.29^{\bullet}$ & 2.74 & 0.13 & 1.23 & 0.11 & 1.00 & 0.16 & 1.48 & 2.00 \\
\hline & 70 & 90 & 88 & $0.27^{\bullet}$ & 2.63 & 0.08 & 0.75 & 0.14 & 1.30 & 0.17 & 1.65 & 2.00 \\
\hline & 80 & 95 & 93 & $0.24^{\bullet}$ & 2.44 & 0.09 & 0.90 & 0.13 & 1.25 & 0.16 & 1.52 & 2.00 \\
\hline & 90 & 100 & 98 & $0.26^{\bullet}$ & 2.66 & 0.06 & 0.58 & 0.11 & 1.13 & 0.14 & 1.40 & 2.00 \\
\hline & 100 & 103 & 101 & $0.24^{\bullet}$ & 2.51 & 0.05 & 0.49 & 0.12 & 1.26 & 0.15 & 1.57 & 2.00 \\
\hline & 120 & 112 & 110 & $0.22^{\bullet}$ & 2.39 & -0.04 & -0.38 & 0.18 & 1.92 & 0.16 & 1.75 & 2.00 \\
\hline & 140 & 118 & 116 & $0.22^{\bullet}$ & 2.44 & $0.02^{\bullet}$ & 0.22 & $0.21^{\bullet}$ & 2.30 & 0.14 & 1.48 & 2.00 \\
\hline & 160 & 121 & 119 & $0.19^{\bullet}$ & 2.13 & 0.07 & 0.74 & 0.12 & 1.30 & 0.15 & 1.65 & 2.00 \\
\hline & 180 & 121 & 119 & 0.14 & 1.59 & 0.12 & 1.28 & 0.13 & 1.46 & 0.14 & 1.57 & 2.00 \\
\hline & 200 & 121 & 119 & 0.14 & 1.52 & 0.13 & 1.44 & 0.13 & 1.45 & 0.15 & 1.69 & 2.00 \\
\hline \multirow{13}{*}{$M \geq 2$} & 30 & 45 & 43 & $0.39^{\bullet}$ & 2.78 & 0.13 & 0.83 & 0.14 & 0.89 & $0.35^{\bullet}$ & 2.46 & 2.02 \\
\hline & 40 & 64 & 62 & $0.36^{\bullet}$ & 3.08 & 0.10 & 0.76 & 0.10 & 0.81 & 0.21 & 1.71 & 2.00 \\
\hline & 50 & 78 & 76 & $0.31^{\bullet}$ & 2.88 & 0.13 & 1.18 & 0.11 & 0.98 & 0.19 & 1.71 & 2.00 \\
\hline & 60 & 86 & 84 & $0.29^{\bullet}$ & 2.73 & 0.14 & 1.33 & 0.11 & 1.03 & 0.16 & 1.51 & 2.00 \\
\hline & 70 & 91 & 89 & $0.26^{\bullet}$ & 2.59 & 0.09 & 0.81 & 0.14 & 1.35 & 0.17 & 1.61 & 2.00 \\
\hline & 80 & 97 & 95 & $0.25^{\bullet}$ & 2.48 & 0.08 & 0.79 & 0.12 & 1.22 & 0.16 & 1.55 & 2.00 \\
\hline & 90 & 101 & 99 & $0.27^{\bullet}$ & 2.76 & 0.04 & 0.38 & 0.11 & 1.10 & 0.14 & 1.42 & 2.00 \\
\hline & 100 & 104 & 102 & $0.25^{\bullet}$ & 2.61 & 0.03 & 0.27 & 0.12 & 1.20 & 0.16 & 1.64 & 2.00 \\
\hline & 120 & 112 & 110 & $0.22^{\bullet}$ & 2.38 & -0.04 & -0.39 & 0.18 & 1.92 & 0.17 & 1.78 & 2.00 \\
\hline & 140 & 119 & 117 & $0.24^{\bullet}$ & 2.63 & 0.02 & 0.17 & 0.19 & 2.10 & 0.14 & 1.54 & 2.00 \\
\hline & 160 & 121 & 119 & $0.19^{\bullet}$ & 2.13 & 0.07 & 0.72 & 0.12 & 1.35 & 0.15 & 1.67 & 2.00 \\
\hline & 180 & 121 & 119 & 0.14 & 1.53 & 0.12 & 1.27 & 0.14 & 1.49 & 0.14 & 1.56 & 2.00 \\
\hline & 200 & 121 & 119 & 0.14 & 1.53 & 0.13 & 1.46 & 0.13 & 1.46 & 0.15 & 1.71 & 2.00 \\
\hline
\end{tabular}


Table 4. Results of the cross-correlation analysis for the short period waves P1, K1 and S2. The index $m$ of $r$ denotes the number of terms matched, which in our case coincides with the number $N$ of $C>0$ in each chain. In addition $N$ must be at least 3 in order to fulfill the condition $n^{*} \geq 3$. The seismicity index $C$ was computed including earthquakes with $M \geq 5, M \geq 4, M \geq 3$ and $M \geq 2$. Correlation coefficients marked by a ${ }^{\bullet}$ are passing the $t$-test for significance level equal to 0.05 .

\begin{tabular}{|c|c|c|c|c|c|c|c|c|c|c|}
\hline \multirow[b]{2}{*}{ Magnitude } & \multirow[b]{2}{*}{$R(\mathrm{~km})$} & \multirow[b]{2}{*}{$m$} & \multirow[b]{2}{*}{$n^{*}-2$} & \multicolumn{2}{|c|}{$\mathrm{P} 1$} & \multicolumn{2}{|c|}{$\mathrm{K} 1$} & \multicolumn{2}{|c|}{$\mathrm{S} 2$} & \multirow[b]{2}{*}{$t_{n^{*}-2}^{0.025}$} \\
\hline & & & & $r_{m}$ & $t$ & $r_{m}$ & $t$ & $r_{m}$ & $t$ & \\
\hline \multirow{13}{*}{$M \geq 5$} & 30 & 1 & - & - & - & - & - & - & - & \\
\hline & 40 & 1 & - & - & - & - & - & - & - & \\
\hline & 50 & 1 & - & - & - & - & - & - & - & \\
\hline & 60 & 2 & - & - & - & - & - & - & - & \\
\hline & 70 & 3 & 1 & 1.00 & 10.36 & 0.93 & 2.62 & 0.99 & 7.63 & 12.71 \\
\hline & 80 & 3 & 1 & 1.00 & 10.36 & 0.93 & 2.62 & 0.99 & 7.63 & 12.71 \\
\hline & 90 & 3 & 1 & 1.00 & 10.36 & 0.93 & 2.62 & 0.99 & 7.63 & 12.71 \\
\hline & 100 & 4 & 2 & 0.82 & 2.02 & 0.82 & 2.00 & $0.98^{\bullet}$ & 7.23 & 4.30 \\
\hline & 120 & 5 & 3 & 0.88 & 3.15 & 0.86 & 2.86 & $0.98^{\bullet}$ & 9.63 & 3.18 \\
\hline & 140 & 6 & 4 & $0.87^{\bullet}$ & 3.61 & $0.85^{\bullet}$ & 3.27 & $0.98^{\bullet}$ & 11.22 & 2.78 \\
\hline & 160 & 12 & 10 & $0.81^{\bullet}$ & 4.40 & $0.72^{\bullet}$ & 3.30 & $0.92^{\bullet}$ & 7.59 & 2.23 \\
\hline & 180 & 13 & 11 & $0.81^{\bullet}$ & 4.40 & $0.72^{\bullet}$ & 3.30 & $0.92^{\bullet}$ & 7.59 & 2.23 \\
\hline & 200 & 13 & 11 & $0.81^{\bullet}$ & 4.40 & $0.72^{\bullet}$ & 3.30 & $0.92^{\bullet}$ & 7.59 & 2.23 \\
\hline \multirow{13}{*}{$M \geq 4$} & 30 & 9 & 7 & 0.61 & 2.02 & $0.69^{\bullet}$ & 2.49 & $0.97^{\bullet}$ & 10.45 & 2.36 \\
\hline & 40 & 17 & 15 & $0.52^{\bullet}$ & 2.36 & $0.63^{\bullet}$ & 3.11 & $0.92^{\bullet}$ & 9.23 & 2.13 \\
\hline & 50 & 19 & 17 & $0.48^{\bullet}$ & 2.27 & $0.58^{\bullet}$ & 2.92 & $0.89^{\bullet}$ & 7.85 & 2.11 \\
\hline & 60 & 25 & 23 & $0.42^{\bullet}$ & 2.23 & $0.46^{\bullet}$ & 2.47 & $0.81^{\bullet}$ & 6.58 & 2.07 \\
\hline & 70 & 30 & 28 & $0.41^{\bullet}$ & 2.37 & $0.42^{\bullet}$ & 2.46 & $0.78^{\bullet}$ & 6.68 & 2.05 \\
\hline & 80 & 32 & 30 & $0.41^{\bullet}$ & 2.48 & $0.42^{\bullet}$ & 2.57 & $0.78^{\bullet}$ & 6.83 & 2.04 \\
\hline & 90 & 35 & 33 & $0.36^{\bullet}$ & 2.21 & $0.37^{\bullet}$ & 2.29 & $0.75^{\bullet}$ & 6.55 & 2.04 \\
\hline & 100 & 38 & 36 & 0.32 & 2.01 & 0.32 & 2.02 & $0.69^{\bullet}$ & 5.75 & 2.04 \\
\hline & 120 & 49 & 47 & 0.26 & 1.87 & 0.26 & 1.87 & $0.63^{\bullet}$ & 5.63 & 2.04 \\
\hline & 140 & 57 & 55 & 0.19 & 1.46 & 0.19 & 1.43 & $0.56^{\bullet}$ & 4.97 & 2.04 \\
\hline & 160 & 72 & 70 & 0.17 & 1.40 & 0.15 & 1.25 & $0.56^{\bullet}$ & 5.70 & 2.00 \\
\hline & 180 & 86 & 84 & 0.13 & 1.21 & 0.12 & 1.06 & $0.50^{\bullet}$ & 5.25 & 2.00 \\
\hline & 200 & 99 & 97 & 0.10 & 1.02 & 0.09 & 0.85 & $0.42^{\bullet}$ & 4.59 & 2.00 \\
\hline \multirow{13}{*}{$M \geq 3$} & 30 & 42 & 40 & 0.28 & 1.85 & 0.30 & 1.97 & $0.65^{\bullet}$ & 5.38 & 2.02 \\
\hline & 40 & 59 & 57 & $0.27^{\bullet}$ & 2.13 & $0.31^{\bullet}$ & 2.44 & $0.61^{\bullet}$ & 5.82 & 2.02 \\
\hline & 50 & 75 & 73 & 0.18 & 1.53 & 0.22 & 1.88 & $0.53^{\bullet}$ & 5.27 & 2.00 \\
\hline & 60 & 84 & 82 & 0.12 & 1.12 & 0.12 & 1.07 & $0.47^{\bullet}$ & 4.78 & 2.00 \\
\hline & 70 & 90 & 88 & 0.15 & 1.41 & 0.16 & 1.52 & $0.46^{\bullet}$ & 4.86 & 2.00 \\
\hline & 80 & 95 & 93 & 0.13 & 1.27 & 0.13 & 1.23 & $0.45^{\bullet}$ & 4.85 & 2.00 \\
\hline & 90 & 100 & 98 & 0.12 & 1.20 & 0.11 & 1.11 & $0.45^{\bullet}$ & 4.96 & 2.00 \\
\hline & 100 & 103 & 101 & 0.10 & 0.96 & 0.11 & 1.08 & $0.43^{\bullet}$ & 4.73 & 2.00 \\
\hline & 120 & 112 & 110 & 0.08 & 0.80 & 0.10 & 1.04 & $0.37^{\bullet}$ & 4.17 & 2.00 \\
\hline & 140 & 118 & 116 & 0.10 & 1.08 & 0.11 & 1.23 & $0.40^{\bullet}$ & 4.68 & 2.00 \\
\hline & 160 & 121 & 119 & 0.09 & 0.95 & 0.08 & 0.92 & $0.40^{\bullet}$ & 4.70 & 2.00 \\
\hline & 180 & 121 & 119 & 0.08 & 0.88 & 0.07 & 0.79 & $0.40^{\bullet}$ & 4.74 & 2.00 \\
\hline & 200 & 121 & 119 & 0.06 & 0.65 & 0.05 & 0.56 & $0.39^{\bullet}$ & 4.59 & 2.00 \\
\hline \multirow{13}{*}{$M \geq 2$} & 30 & 45 & 43 & 0.29 & 1.95 & $0.30^{\bullet}$ & 2.06 & $0.65^{\bullet}$ & 5.54 & 2.02 \\
\hline & 40 & 64 & 62 & $0.25^{\bullet}$ & 1.99 & $0.28^{\bullet}$ & 2.32 & $0.54^{\bullet}$ & 5.05 & 2.00 \\
\hline & 50 & 78 & 76 & 0.16 & 1.45 & 0.22 & 1.95 & $0.50^{\bullet}$ & 5.02 & 2.00 \\
\hline & 60 & 86 & 84 & 0.10 & 0.95 & 0.12 & 1.06 & $0.45^{\bullet}$ & 4.56 & 2.00 \\
\hline & 70 & 91 & 89 & 0.14 & 1.35 & 0.16 & 1.54 & $0.47^{\bullet}$ & 5.02 & 2.00 \\
\hline & 80 & 97 & 95 & 0.11 & 1.11 & 0.13 & 1.29 & $0.46^{\bullet}$ & 5.08 & 2.00 \\
\hline & 90 & 101 & 99 & 0.12 & 1.16 & 0.12 & 1.19 & $0.45^{\bullet}$ & 5.07 & 2.00 \\
\hline & 100 & 104 & 102 & 0.09 & 0.90 & 0.11 & 1.13 & $0.43^{\bullet}$ & 4.82 & 2.00 \\
\hline & 120 & 112 & 110 & 0.08 & 0.82 & 0.10 & 1.05 & $0.37^{\bullet}$ & 4.19 & 2.00 \\
\hline & 140 & 119 & 117 & 0.09 & 1.00 & 0.09 & 1.03 & $0.38^{\bullet}$ & 4.50 & 2.00 \\
\hline & 160 & 121 & 119 & 0.09 & 0.96 & 0.09 & 0.95 & $0.40^{\bullet}$ & 4.74 & 2.00 \\
\hline & 180 & 121 & 119 & 0.08 & 0.89 & 0.07 & 0.78 & $0.40^{\bullet}$ & 4.73 & 2.00 \\
\hline & 200 & 121 & 119 & 0.06 & 0.63 & 0.05 & 0.57 & $0.39^{\bullet}$ & 4.62 & 2.00 \\
\hline
\end{tabular}


Our results may be considered to be consistent, or at least not contradicting with the general concept of the mechanisms of preseismic phenomena in the atmosphere and ionosphere discussed in Sect. 1. According to this concept precursory phenomena such as atmospheric perturbations of temperature, density etc., result from an upward migration of fluid substrate matter in the strength-weakened areas of the crust.

Acknowledgements. We are very thankful for the important comments by the reviewers, helping us to improve the paper.

Edited by: M. Contadakis

Reviewed by: P. F. Biagi and another anonymous referee

\section{References}

Arabelos, D., Asteriadis, G., Contadakis, M. E., Spatalas, S. D., and Sachsamanoglou, H.: Atmospheric tides in the area of Thessaloniki, J. Geodyn., 23, 65-75, 1997.

Arabelos, D., Asteriadis, G., Bloutsos, A., Contadakis, M. E., Kaltsikis, Ch., and Spatalas, S. D.: Atmospheric tides in the area of Thessaloniki-North Greece. A twenty-seven years data analysis, From Stars to Earth and Culture, In honour of the memory of Professor Alexandros Tsioumis, edited by: Dermanis, A., Publishing Ziti, Thessaloniki, 201-207, 2003.

Arabelos, D. N., Asteriadis, G., Bloutsos, A., Contadakis, M. E., and Spatalas, S. D.: Atmospheric tide disturbances as Earthquake precursory phenomena, Nat. Hazards Earth Syst. Sci., 4, 1-7, 2004, http://www.nat-hazards-earth-syst-sci.net/4/1/2004/.

Bartzokas, A.: Dynamical factors influencing the daily barometric fluctuation in the vicinity of the ground, in the area of Greece, Ph.D. Thesis, University of Ioannena, Greece, 208 pp., 1989.

Bartzokas, A., Repapis, C. C., and Metaxas, D. A.: Temporal variations of atmospheric tides over Athens, Meteorol. Atmos. Phys., 55, 113-123, 1995.

Biagi, P. F., Piccolo, R., Capozzi, V., Ermini, A., Martellucci, S., and Bellecci, C.: Exalting in atmospheric tides as earthquake precursor, Nat. Hazards Earth Syst. Sci., 3, 197-201, 2003, http://www.nat-hazards-earth-syst-sci.net/3/197/2003/.

Chapman, S. and Lindzen, R. S.: Atmospheric Tides, DortrechtHolland: D. Reidel, 200 pp., 1970.

Choy, G. L. and Boatwright, J. L.: Global patterns of radiated seismic energy and apparent stress, J. Geophys. Res., 100(B9), 18 205-18 228, 1995.

Davis, J. C.: Statistics and data Analysis in Geology, John Wiley and Sons, Inc., New York, London, Sydney, Toronto, 241-246, 1973.
Gorbatikov, A. V., Kodama, T., Molchanov, O. A., and Hayakawa, M.: Long period variations in seismic and electromagnetic measurements, in: Atmospheric and Ionospheric Electromagnetic Phenomena Associated with Earthquakes, edited by: Hayakawa, M., Terra Sci. Publ. Comp., Tokyo, 439-450, 1999.

Gutenberg, B. and Richter, C. F.: The energy of earthquakes, Q. J. Geol. Soc. London, 112, 1-14, 1956.

Hayakawa, M., Molchanov, O. A., Ondoh, T., and Kawai, E.: The precursory signature effect of the Kobe earthquake in VLF subionospheric signal, J. Comm. Res. Lab., Tokyo, 43, 169-180, 1996.

Hayakawa, M. (Ed.): Atmospheric and Ionospheric Phenomena Associated with Earthquakes, Terrapub, Tokyo, 996 pp., 1999.

Hayakawa, M. and Molchanov, O. A. (Eds.): Seismo Electromagnetics, Lithosphere-Atmosphere-Ionosphere Coupling, Terrapub, Tokyo, 477 pp., 2002.

Mareev, E. A., Iudin, D. I., and Molchanov, O. A.: Mosaic source of internal gravity waves associated with seismic activity, in: Seismo-Electromagnetics (lithosphere-athmosphereionosphere coupling), edited by: Hayakawa, M. and Molchanov, O., Terrapub, Tokyo, 335-342, 2002.

Molchanov, O., Schekotov, A., Fedorov, E., Belyaev, G., and Gordeev, E.: Preseismic ULF electromagnetic effect from observation at Kamchatka, Nat. Hazards Earth Syst. Sci., 3, 203-209, 2003, http://www.nat-hazards-earth-syst-sci.net/3/203/2003/.

Molchanov, O., Fedorov, E., Schekotov, A., Gordeev, E., Chebrov, V., Surkov, V., Rozhnoi, A., Andreevsky, S., Iudin, D., Yunga, S., Lutikov, A., Hayakawa, M., and Biagi, P. F.: Lithosphereatmosphere-ionosphere coupling as governing mechanism for preseismic short-term events in atmosphere and ionosphere, Nat. Hazards Earth Syst. Sci., 4, 757-767, 2004, http://www.nat-hazards-earth-syst-sci.net/4/757/2004/.

Plotkin, V. V.: GPS detection of ionospheric perturbation before the 13 February 2001, El Salvador earthquake, Nat. Hazards Earth Syst. Sci., 3, 249-253, 2003, http://www.nat-hazards-earth-syst-sci.net/3/249/2003/.

Rikitake, T. (Ed.): Current Research in Earthquake Prediction, vol. I, Developments in Earth and Planetary Sciences, D. Reidel, London, 1-80, 1981.

Silina, A. S., Liperovskaya, E. V., Liperovsky, V. A., and Meister, C.-V.: Ionospheric phenomena before strong earthquakes, Nat. Hazards Earth Syst. Sci., 1, 113-118, 2001, http://www.nat-hazards-earth-syst-sci.net/1/113/2001/.

Tamura, Y.: A harmonic development of the tide generating potential, Bulletin d'Information Marées Terrestres, 99, 6813-6855, 1987.

Tzanis, A. and Vallianatos, F.: Distributed power-law seismicity changes and crustal deformation in the SW Hellenic ARC, Nat. Hazards Earth Syst. Sci., 3, 179-195, 2003, http://www.nat-hazards-earth-syst-sci.net/3/179/2003/. 